\title{
EXPERIENCIA DE LA INVESTIGACION TURISTICA EN VENEZUELA
}

\begin{abstract}
Alfredo Ascanio'
RESUMO: Analisa a pesquisa venezuelana em Turismo nas universidades que sededicam à administração decursos de pós-graduação em planejamento integral e gerenciamento turistico. Apresenta as bases institucionais do Turismo na Venezuela, e enfoca a produção desses cursos destacando: tema e unidades produtoras, novos marcos referenciais, estratégia, táticas de abordagem e natureza. Refere-se, ainda, ao novo modelo de urbanismo turistico aplicado em recentes trabalhos espanhóis, como o plano urbanistico da Costa de Calviá (Mallorca). Conclui sobre a necessidade de relacionamento entre universidade - setorpúblico - setor privado, para a realização de um programa coerente e prioritário sobre as principais pesquisas em Turismo a serem desenvolvidas.

PALAVRAS-CHAVE: Pesquisa em turismo; cursos de pós-graduação; produção acadêmica; Venezuela.

ABSTRACT: This paper analy'sis the venezuelan research in Tourism carried out at the universities ministrating postgraluate courses in Tourism integral planning and Tourism management. It presents the institutional basis of Tourism in Venezuela, and focuses on the issues of these courses stressing: subject and production departments, new frames of reference, strategies, approach tactics and nature. It refers, also, to the new urban model fortourism set up in recent Spanish plans, like the urbanistic plan of Costa de Calvia Mallorca). It concludes that relationship between university', public sector and private sector is needed, to establish a coherent and priority-oriented research program to be developed.
\end{abstract}

KEY WORDS: Tourism research; postgraduate courses, academic production, Venezuela.

1 Economista e pós-graduado em Harvard. Professor da Universidade Simón Bolivar, Caracas, Venezuela. Ex-assessor de organismos internacionais de Turismo.

End. para corresp.: Urb. Alto Prado - Calle 10 y Av. 12 - Qta. Los Helechos - Caracas 1080 Venezuela. 


\section{UNA BREVE INTRODUCCION}

El turismo en América Latina. y en Venezuela en particular. es una actividad muy reciente. A finales del año 1936 se redactó en Venezuela el primer Anteproyecto de la Ley de Turismo, que luego es promulgada en 1938. Es justo scriala quefue en la Revista del Ministerio de fomento de aquel año 38. cuando por primera vezse publicanarticulosquehacen referencia a la actividad turistica, con cl objeto de cstablecer criterios y concientizar a los entes gubernamentales sobre la importancia cconómica de esta actividad de sen'icios.

Los planes para el Fomento del Turismo en Venezucla, tienen su antecedente más remoto en los documentos presentados por la Dirección de Industrias del Ministerio de Fomento, cn cl año de 1947 (CÁCERES, 199(): +(0) y luego Ic tocará a la Dirección Nacional de Turismo, para el año de 1953, realizar las primeras investigaciones descriptivas, a los fines prácticos de la promoción en el Exterior. conjuntamente con firmas especializadas de la ciudad de $\mathrm{New}$ York

Postcriormentc, la Corporación Nacional de Hoteles y Turismo (CONAHOTU), se dedica básicamente a la actividad de gerencia y operación hotelcra, lo cual interrumpe los primeros esfuerzos en conocer con datos estadísticos el desarrollo turístico venczolano, pues el Estado se interesó mayormente por ser un empresario hotelero y por cllo dedica pocos esfuerzos al diagnóstico y a la gencración de políticas turísticas.

La recesión turistica del periodo 1959-1962 no es investigada y sólo sirve para tomar la decisión de declarar al turismo como una actividad de interćs público. Más tarde, la Junta de Fomento Turístico promueve la preparación del Plan Nacional de Turismo y los Planes Regionales; y en especial, el Plan Nacional realizado en 1969) con la colaboración técnica del Gobicrno ltaliano! la participación de la OEA

En ese año de 1969) es la primera vez que, de una manera sistemática. se realiza un inventario de los recursos turísticos: y se llevan a cabo estudios estadisticos comparativos de los datos referidos al viaje temporal.

El Plan anterior coicide con la década de los años 7(), cuando se inician los primeros esfuerzos organizados de una acción gubernamental en materia de turismo; y cuando aparecen, en el IV Plan de la Nación (197()-1974), criterios más claros para conocer bien el sector, integrándose el turismo al sistema de planificación nacional.

En el año de 1972. la Gerencia de Operaciones Regionales de la Corporación de Turismo de Venezucla. Dirección de Planificación. pública miás de 20) documentos técnicos, donde se diagnostican las estnicturas receptivas de los 2() Estados y los 2 Territorios Federales
Con la Ley de Turismo de 1973, se impulsan los estudios que sobre el Con territorial turístico y se realizan los primeros planes de zonas ordenanuento territor como, por cximplo: el cstudio sobre la zona costera Pucrto La Cruz turisticaś: el estudio turístico de los Caracas-Higucrote, realizado con cl Banco Mundial; la Faja Costera desde Punta-Castillete, en el Zulia; cl cstudio costero Mundaal; la Faja Co Paraguaná. en el llamada Región Centro Occidental; cl de la Pe costero Tucacas-Chichirivichc; el ordenamiento territorial turistico de estudio costero Tarita; cl plan rectordel Parque Nacional Canaima, realizado con la colaboración y asistencia del Senicio de Parques del Departamento del Interior de los Estados Unidos del Norte.

En el año de 1978. La Olicina de Planificación del Ministerio de Información y Turismo publica, en más de 12 volúmenes, el Inventario Completo de la Ojerta liuristica Nacional y' se realizan los primeros estudios sobre patrimonio socio-cultural y artesanal del Pais.

Cuando se promulga la Leý Orgánica para la Ordenación del Territorio en 1983, alli se establecen las grandes directrices para armonizar los usos del suelo turístico y para que su control se realice en régimen de Administración Especial.

EnelPlan de Turismo 1984-1989) se hace un análisis de la política turistica y se redefine la actividad turística venezolana: y en el VIII Plan de la Nación (1989-1993), se establece que la politica sectorial, en materia de turismo, debe enmarcarse en una estrategia amplia de competitividad a nivel internacional; y por primera vez se mencionan la palabras planificación integral, turismo de aventura en el medio natural y turismo de convenciones.

Al crearse en Venezucla el Consejo Nacional de Promoción de Inversiones (CONAPRI) y el Comité de Turismo de la Cámara Venezolano-Americana de Comercio e Industria (VENAMCHAM), se realizan, desde 199(), varias publicaciones sobrc oportunidades de inversión turistica en el pais de los autores David LeRoy Foote y Donald E. Hawkins; c incluso, algunos números de la revista de la Cámara, denominada Business l'enezuela, se han dedicado a resumenes completos de las cstadisticasdel sector (n. 145, 1992). La publicación de CONAPRI, denominada una (illia para el Inversionista en Tiurismo (nov: 1992), publica las estadisticas más recientes y asi como las leves y reglamentos que tienen relación con la actividad turística de Venczuela (Investigación de los autores Eduardo Carrasquero, Omar Vera, Mariscla Ascanioy Alfredo Ascanio)

El foro realizado por el Instituto de Estudios Superiores de Administración (IESA) denominado: "Turismo Algo Más que un Charter" es un primer intento valioso de rescatar la investigación básica para orientar a la gerencia turística del pais, al igual que otros foros realizados durante las décadas de los años 8()$^{9} 9()$

Como ustedes ven, el csfuerzode la planificación turistica venezolana se ha realizado sin contar previamente con un buen sistema estadístico sobre csta 
importante actividad; y sin una estrategia para estimular la investigación bisicia y aplicada en aquellas instituciones, que como las Universidades, tienen el perfil adecuado para desarrollar esta importante tarea que además le sirve como apovo a su actividad docente y especialmente para la divulgación del conocimiento.

\section{EL ROL DE LA UNIVERSIDAD}

Cuando surgen las Maestrías de Turismo en Venezuela, con las primeras iniciativas del Servicio Económico Latinoamericano (SELA), es cuando se estimulan los estudios de post-grado en turismo y cuando se empiczinl a desarrollar, de una manera sistemática, los trabajos de grados en estecampo. que en su gran mayoría responden a la necesidad de contar con planes turísticos tanto a escala regional como a escala distrital y estudios de circuítos turísticos nacionales.

Entre 1986 y 1992 por ejemplo, en los Talleres de la Maestría sobre la Planificación Integral para el Desarollo del Turismo de la Universidad del Zulia, se llevan a cabo más de 11 planes integrales de turismo y además 3 estudios sobre circuitos turísticos y 2 estudios sobre municipios turísticos, una actividad de investigación descriptiva muy valiosa que debería servir a los gobiernos locales para implementar programas turísticos novedosos.

Estos estudios se analizan bajo una óptica diagnóstica y tomandoen cuenta los elementos geográficos, físico-espacial, económico, social y político institucional. Los estudios tienen como objetivo central la proposición de lineamientos generales de acción en subprogramas, con sus estrategias de implementación.

A veces los estudios se orientan hacia el establecimiento de metodologías de trabajo para ordenar un recurso turístico determinado; o bien para estudiar un espacio turístico, su funcionamiento y su posible utilización racional

Se han realizado estudios estratégicos para el aprovechamiento integral de circuítos turísticos; o bien estudios sobre la reglamentación de corredores turísticos de traslado, partiendo de los criterios referidos a la planificación integral.

Se han elaborado también tesis de grado sobre el turismo urbano en centros de interés histórico, como el Empedrao, en Maracaibo, con el objetivo de establecer una estrategia de acción.

Igualmente, la planifición estratégica situacional y a nivel municipal. a fin de definir políticas y acciones turísticas locales, ha sido uno de los temas tratados en la Maestría de Turismo de la Facultad de Arquitectura de la Universidad del Zulia (LUZ).

En lo que se refiere a los trabajos de los estudiantes del post-grado de Turismo, en la Facultad e Ciencias Económicas y Sociales de LUZ, se han mollado más de una decena de investigaciones sobre diversos temas, como: esarro fronterizo de la Zona Noroccidental del Estado Zulia; modelos de el turismo y conservación, utilizando como medio al turismo rural; uso de desarrollo y conservacion, con fines turísticos; necesidades de adiestramiento infraestruch hotelera del Estado Zulia; turismo religioso en el Estado Trujillo; para el área hós histórica del turismo en Venezuela; geografia turística del Estado la evolución historica de turismo para lugares puntuales de los Estados Falcón, Trujillo y Aragua; y, modelos de gestión para empresas turísticas

Estebreve recuento de los trabajos de investigación, en materia de turismo, la Universidad del Zulia, es un buen cjemplo de un trabajo pionero en materia de planificación integral y de gestión turística.

En otras Maestrías de Turismo que existen en Venezuela, como la Maestría de Turismo de la Universidad Francisco de Miranda (UNEFM) en Coroy del Núcleo en Margarita, de la Universidad de Oriente, se han realizado trabajos similares; al igual que otras escuelas y facultades universitarias como las tesis de grado en el Núcleo Universitario (NUL) de la USB, la Coordinación de Urbanismo de la Universidad Simón Bolivar y trabajos en la Cátedra de Diseño de la Facultad de Arquitectura y Urbanismo de la Universidad Centra de Venezuela, asi comolas publicaciones sobre turismo de la Revista Investigación \& Gerencia.

\section{NUEVOS MARCOS REFERENCIALES}

La investigación en turismo, como todo trabajo especializado, ha surgido porque existe, en forma embrionaria, un marco teórico o referencial que le sirve de soporte. No obstante, a su vez hacer investigaciónen turismo supone descubrir nuevos tópicos de estudio, que requieren de perspectivas teóricas novedosas y de procedimientos metodológicos innovadores (DANN, et al., 1988: 2).

Es apenas en el año de 1970, cuando se comienza a legitimar, en algunas universidades del mundo, al turismo como un área para investigaciones sistemáticas (ASCANIO, 1992: 190). En Venezucla legitimamos en la Academia esta actividad, diez años más tarde lo cual es un desfase en el tiempo bastante grande.

Como vimos en el resumen de los párrafos anteriores, el turismo existió institucionalmente, en nuestro país, desde 1938; pero fue apenas en la década de los años 80 , cuando las universidades comienzan a realizar estudios de postgrados y tesis te maestría. Con todo, aún existen muchas dificultades para consolidar una tarea que consideramos relevante.

El investigador que se dedica al área del turismo, y en especial, la planificación integral, debe comenzar a reconocer varios asuntos que en otros 
países ya se señalan como nuevos elementos que van a permitir establecer una nueva teoría del urbanismo turístico (LEIRA, et al., 199 la: 2-11), como son:

a) el turismo no debe basarse solamente en una actividad urbanizadora constructora, como sí se estuviera planificando una ciudad. El turismo requiere de un modelo propio que, a diferencia de las ciudades industriales, no privilegie la producción con carácter mercantil de plazas de alojamiento masivas y concentradas en una franja de litoral, que con el tiempo se transforman en zonas saturadas y en "productos obsoletos" o envejecidos en forma prematura;

b) el turismo requiere de un reparto espacial, o de un modelo territorial más adecuado al consumo turístico, desde una perspectiva regional y donde el asunto de la rentabilidad del sector no se realice con intensidades de uso de los espacios, sino porque se le "añade valor" a zonas potenciales que no deben ser modificadas, al extremo, en su contexto natural y socio-cultural;

c) la planificación del turismo debe darle mayor peso a la calidad en relación a la cantidad. La teoría y la práctica de la arquitectura, del urbanismo y del paisajismo como disciplinas, para formular el problema turístico, no pueden servir ellas solas como fuentes para planificar un espacio natural. Las propuestas que se derivan del racionalismo de urbanizar, como si se tratara de ciudades, ha contribuido, en parte, a cxpoliar el paisaje que le dio origen y que se considera un patrimonio que debe ser conservado;

d) el investigador debe entender las variables esenciales que definen el proceso de proyectación del suelo y del paisaje. El problema es que las variables son muchas, pues el turismo es multidiciplinario y en ello consiste la dificultad de la investigación en este campo. Y la dificultad es mayor, cuando se toman en cuenta los aspectos socio-culturales y sus impactos debido a su naturaleza de orden cualitativo.

\section{LA ESTRATEGIA Y LAS TACTICAS PARA LA \\ INVESTIGACION}

Siempre se nos ha dicho que la estrategia de la investigación consiste en aplicar el método científico y que cada tema a ser investigado, tiene su propia naturaleza y un estado del conocimiento dado (BUNGE, 1980: 31 ). Así. por ejemplo, la determinación de un plan coherente del suelo turístico exige una técnica integral y sistemática, requerida para descubrir la relación entre el espacio y sus usuarios potenciales. La resolución efectiva del primer problema. dependerá del estado en que se encuentre la teoría del urbanismo turístico, igual que la resolución del segundo problema dependerá del estadoen que se encuentrc la teoría del comportamiento del turista, y la población de acogida, en los espacio recreativos; la tcoría de las percepciones del ambiente y otras disciplinas pertinentes.

Cada disciplina que interviene en la planificación del suelo plantea una a ca técnica propia (un instrumento de análisis), pero la estrategia es una tactica o lectiar el trabajo en base al método científico, es decir:

a) enunciar bien el problema:

b) arbitrar conjeturas que se puedan contrastar con la experiencia

b) arbitrar consequencias lógicas de las conjeturas:

c) derivar consequencias a contrastación, así como las técnicas utilizadas:

d) someter las los resultados

e) estimar la fidelidad de los investigado;

g) determinar los domínios en los cuales valen las conjeturas y las técnicas, para poder formular mucros conocimientos y problemas.

\section{LA NATURALEZA DE LA INVESTIGACION TURISTICA}

En cuanto a la naturaleza del turismo para ser investigado, Clare Gunn, de la Universidad de Texas, nos señala que dada la variedad de las disciplinas que intervienen en él. es frecuente que sea un grupo multidisciplinario de investigadores los que se dediquen a esta tarea (GUNN, 1987: 6).

Como el turismo no tiene una larga historia, en cuanto a investigaciones se refiere, todavía los estudios son descriptivos y exploratorios; no obstante, ellos nos proporcionan nuevos datos y enfoques que nos ayuden a pasar a otra etapa más importante como seria la investigación explicativa o bien la evaluativa.

Aunque existen una gran variedad de investigaciones y técnicas de análisis, lo cual puede llevarnos a la confusión, la naturaleza de la investigación en turismo - scgún mi opinión - debe ser aquella que permita tomar mejores decisiones al contribuir al diseño de las políticas públicas y de la política a nível de la gerencia turística. A este fin, la categoría de los tipos de investigación sugeridos por Brent Ritchie, de la Universidad de Calgary, Canadá, nos ayudarian a seleccionar un enfoque adecuado a nivel de la Academía:

a) investigación operacional, que utiliza técnicas cuantitativas y analíticas, nos permitiriría formular decisiones para optimizar la relación entre insumos y productos. Un ejemplo: determinar el tráfico óptimo entre origen y destinos turisticos;

b) Investigación gerencial, en la búsqueda de soluciones puntuales para ser implementadas a corto plazo. Un cjemplo: el estudio de factibilidad para un nuevo hotel; 
c) investigación estratégica, que se puede dividir en investigación sobre politicas y actividades de la planificación integral (investigación sistémica). e investigaciones para realizar el seguimiento después de implementar politicas o planes (evaluaciones ex-post).

La clasificación anterior nos indica, que la Academía puede mantener una relación universidad-mundo exterior, a través de sus investigaciones, para contribuir con el proceso de la toma de decisiones públicas y privadas. De esta forma se puede contribuir con una investigación que cumpla la función de mejorar la formulación de políticas y la toma de decisiones en el campo del turismo.

Este enfoque, de una investigación universitaria aplicada al mundo real. con vistas al domínio de un conocimiento bien fundado (Episteme), ha sido apoyada en Estados Unidos por David Edgell, Director de la Oficina de Politica y Planificación del Turismo, y por Jay Beaman, Director de los Programas de Gerencia del Servicio de Parques del Canada.

No obstante, para que el trabajo de investigación, que se derive de los trabajos de las maestrias de turismo, sean éectivos y útiles al mundo público y privado, se requieren unas nuevas condiciones de organización a nível de la Universidad, y sus escuclas de post-grados turísticos, como:

a) un soporte gerencial de tutores-especialistas, que puedan dirigir las investigaciones, apoyadas en Unidades de Coordinación entre el programa docente de post-grado en turismo y la investigación. Unidades Coordinadoras que puedan operar con autonomía en el análisis de temas importantes, y con carácter prospectivo, como algunos que señalamos en este trabajo.

b) un suficiente apoyo al estudiante de las maestrias, en cuanto a matcrial bibliográfico se refiere; trabajos de campo y seminarios de discusión

c) un canal de comunicación ágil entre universidad y sector público y/o privado. a fin de buscar contratos de investigación específicas con un financiamiento asegurado;

d) la posibilidad de divulgar los hallazgos para que se puedan implementar las soluciones encontradas. (BEAMAN, 1987: 42).

La fase de ayuda en la implementación y seguimiento de los planes ! proyectos de investigación es clave, por dos razones: porque como lo señaló. un vez, BACAL (1991: 108),

$$
\text { es má fácil poner en evidencia los cambios conómicos mensurables que los }
$$$$
\text { efectos socio-culturales cralitatuvo. }
$$

y porque un estudio que no se sabe " vender luego al cliente para que lo ponga préctica es un estudio que va a los anaqueles y con él se pierde esfuerzo, dinero posibilidades de cambio.

Para terminar, me voy a referir al nuevo modelo de urbanismo turístico we se haesto en práctica en algumos desarrollos recientes españoles, como el Plan Urbanístico y Concertado para el Desarrollo Turístico de la Costa de Calviá, en Mallorca.

\section{EL MODELO DE URBANISMO TURISTICO Y LA CALIDAD \\ DEL SUELO TURISTICO}

El desarrollo de las ciudades y la urbanización turística no son fenómenos de naturaleza análoga entre sí; por lo tanto. no se puede aplicar a la organización y al proyecto de los espacios turisticos, las mismas nociones y métodos experimentados en las ciudades. El desarrollo turístico es bien diferenciado y debe tener sus propios conceptos e instrumentos para investigarlo.

La ciudad es cosmopolita y genera su propio modo de comunicación social. La ciudad es plural e integra unidades residenciales. La unidad vecina se logra en la ciudad en su conjunto, con espacios y usos comunes que sirven de soldadura entre las partes. La ciudad se desarrolla con una continuidad incrcial y con una cxpansión en saltos discretos

¿Peroque ocurrecuando se trata de la ocupación de territorios cuyos rasgo de identidad y referencias formales son prístinos y naturales y donde existe, por lo general, una comunidad receptora con sus propios valores socio-culturales bastante frágiles?

La organización territorial del turismo plantea las siguientes exigencias

a) un orden territorial integrado escenográficamente en el espacio natural;

b) el carácter y la función de cada una de las partes o núcleos es diverso y diferenciado;

c) cada pieza del conjunto reclama un carácter autónomo para ser desarrollado:

d) en los núcleos turísticos los visitantes, e incluso los residentes, no residen sino se alojan, lo cual implica que el argumento principal para la ordenación y el uso social de estas áreas no sea "la vivienda", sino las actividades recreativas, locual nos indica que lo que debe predominar es el equipamiento pues el alojamiento pasa a ser un medio necesario para mantener las actividades de recreo y esparcimiento.

Se debe entender así, que el espacio turístico ha de ser usado, vivido, figurado arquitectónicamente y sentido de modo diferente a la ciudad cotidiana Los turistas proyectan en estos espacio otras fantasías y otros comportamientos: 
y' por cllo, el espacio se debe organizar de otra manera para que se respele cl cntorno $y$ a la comunidad de acogida.

Además, el urbanismo turístico no admite un modo de crecinniemno incrcial, porque una implantación turistica no es un barrio, no es una urbanizilión residencial. Esta implantación es un "producto" destinado a satisfacer miá demanda muy articulada y organizada, donde cxistirán otros "produclos" compitiendo por adecuarse a la fantasía del visitante, pero lamentablemente ignorando lo que ocurre en el entorno.

El crecimiento lurístico no se da por piezals urbanas de crecimlento contínuo, pues lo que aparecen son siltos a cscala, donde se agregan piczas sin contiguidad debido a que cada espacio tiene su propio motivo escenogrittico. segúncl modelo recreativo quele da origen. Osca, cada pièa es una escenogralia distinta a las demás.

Si se admiten los criterios anteriores como hipótesis de trabajo, el modelo de urbanizar el espacio turístico debe ser singular y no masivo. No se tralla de asegurar un conglomerado de "camas", plazas turísticas, para of recer luego sol y playa u otro recurso natural. Se trata de cliversificar v calificar la ofe eta. s además, se trata de conciliar el urbanismo furistico con cl paisajey con el entorno socio-cultural, en una moderna economía de senvicios, donde quede bien regulada la capacidad de carga y la calidad del proceso de urbanización

En esta tarea la responsabilidad del sector público consiste en eslablecer las "reglas del juego", pero es al sector privado que le incumbe asegurat un desarrollo turístico ordenado, sustentable y significativo para los turislias. asi como para la comunidad anfitriona.

\section{CONCLUSION}

Hastacl momento la investigaciónturistica en Venczucla se ha desano otlado a nivel de las Universidades quese dedican a la administración de cursos de poslgrados en planificación integral y' gerencia turística.

Aun el Estado Venezolano no ha jugado un rol importante en esta alliat y sinembargo, para poder establecer un conjunto de politicas turistas de significación y una estrategia de desarrollo del sector, se requiere un cucrno de datos estadísticos bien elaborados y un plan de investigaciones que sinva de basc para la toma de decisiones acertadas.

La Universidad está llamada a cumplir este rol de investigar para ifue li sociedad Venezolana realice una mejor gestión turistica.

Se debe estimular un plan Universidad-Sector Público-Sector Pric ado. al los fines de realizar un programa coherente y prioritario sobre las principalcs investigaciones en turismo que permitan crear un cuerpo de politicas ! misticils integrales.
La inrestigación luristica por su propia naturaleza debe ser midisciplinaria. por lo que algunos trabajos de grados se podrían llev'ar a multidiscipliarias Facullades de una Universidad, si esto fuese posible. Incluso. cabo añadir a los csludios una asignalura sobre Técnicas de Análisis en se debe siguiendo el texto docente del prof. Stephen Smith denominado Turismo, signis Ansis (A Handbook)", 1989, con lecturas dirigidas utilizando las 12 principalcs revislas especializadas, a siber: "Estudios Turísticos": "Estudios y Perspecliatas en Turismo": "Annals of Tourism Rescarch": "Tourism Management": "The Tourist Review": "Journal of Travel Rescarch": "The Journal of Tourism Studies": "International Journal of Hospitality Management": "Cornell Hotel and Restaurant Quaterly": "International Tourism Quaterly": "Travel, Tourism and Hospitality Rescarch" " “Contcmporany Hospitality".

En cuanto a las principales lineas de investigación, se deberian estimular las siguientes, de una lista que podría ser mucho más larga según los problemas quese comicnzan adelectarỵ según la visión prospectiva que los expertos tengan del turismo:

a) investigación operacional: modelo para el control de la promoción turística cu el Extcrior; estructura de costos en hotelaria y' su relación con el punto de cquilibrio en un cntorno infacionario; modelo para predecir cambios competitivos en el liempo, en base a la mezcla hoteleria y extrahotelería y' si participación en el mercado; innuencia del tour-operador en la oferta turística y los desbalances entre número de plazis, demanda y tarifas;

b) investigación gerencial: evaluación del riesgo del hotel según diversos grados de apalancanniento financicro; evaluación del mercado turístico internacional cnuna unidad hotelera v's. el turismonacional y la planificación de las utilidades: comportamiento del ecoturista durante los viajes para la observación de ares en dos entornos diferentes y una estrategia del guía para el plan de mancjo:

c) investigación éstratégica: planificación física vs. planificación económica el equilibrio que estimula la inversión; planificación lĩsica integral y su impacto en el medio natural. a los fines de asegurar un punto óptimo de ren

turístico y un nuero enfoque para un desarrollo sustentable y de largo plazo urbanismo turístico y los impactos socio-culturales - in modelo para aminorar los costos sociales: análisıs e as opmmones comunitarias sobre los cambios de los patrones socio-culturales después que irnumpió cl turismo comportamiento recreacional del venczolano $\mathrm{y}$ los problemas de la contaminació visua : isenoturıstıco en el medio nural utilizando materiales naturales autóc onos como el adobe cocido ý el bambú: posibilidades para el 
lurismo de aventura y' el ccolurismo; la Casa Paraguanera o Casas de Hato. posibilidades de su uso turistico para el mercado de Aruba y las Islias Ncherlandesis.

\section{BIBLIOGRAFIA}

ASCANIO, Alfredo. 1992. Turismo: la ciencia social de los viajes. Estudios y, Perspectivats en Turismo. Buenos Aires, v. I, n. 3.

BACAL, Saralı S. 199I. Impacto del turismo en núcleos receptores de paises en desarrollo. Estuchos $y$ Perspectivas en Tiurismo. Buenos Aires, v. 1, n. 2.

BEAMAN, J. 1987. Managing the research function for elfective policy formulation and decision making. Travel, Tourism, and Hospitality' Research. New Y'ork, p. 35-42. (A Ilandbook fier Managers and Rescarcles).

BUNGE, Mario. 1980. La investigación cientifica. Barcelona: Ariel.

CACLRES, R. Juan. 1990. La evolución histórica de la actividad turistica en léenezuidu Alaracaibo: Universidad del Zulia - Facultad de Ciencias Economicas y Sociales. (Tesis de Macstria).

DANN, Graham et al. 1988. Methodology in tourism research. Annals of Tourism Research, v. 15. n. 2.

EDGELL, David L. 1987. The formulation of tourism policy - A managerial lramework. Travel. Tourism, and Hospitality' Research. New Y'ork, p. 22-3.

GUNN, Clare A. A perspective on the purpose and nature of tourism research methods. Travel. Tourism, and Hospitality' Research. New Y'ork.

LEIRA, Eduardo et al. 199 la. Ora práctica de la urbanización turistica. Gieometria, n.12, p. 2-11. . 19916. Un plan urbanistico y concertado para el desarrollo turistico de Calvii (Mallorcil). Geometria, n. 2, p. 54-63.

RITCIIIE, J. R. Brent. 1987. Roles of research in tourism management. Travel, Tourism, and Ilospitality Research. New York.

TURISMO en Venezuela. 1991-92. Caracas: Venam-Cham.

TOURISM in Venezuela: an investor's guide. 1992. Caracas: Conapri.

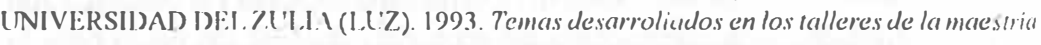
en planificación integral para el desarrollo del turismo. Facultad de Arquitectura. División de Estudios para Graduados (1986-92)

UNIVERSIDAD DEL ZULIA (LUZ). 1993. Trabajos en administración de empresas turisticas. Facultad de Ciencias Económicas y Sociales. División de Estudios para Graduados (1986-88).

(INIVERSIDAD NACIONAL, EAPERIMIENTAL, IRANCISC() DE MIRANDA. 19'. Trabajos de grado. Coordinador de posigrado.

(NIVERSIDAD DEORIENTE: 1093. Trahajos de grado Coordimaciónde postgrado enturistrta
Tabla 1 - Número de trabajos de investigación por año

\begin{tabular}{|c|c|c|c|c|c|}
\hline \multirow{2}{*}{ ANOO } & $1.1 \%, 1 \mathrm{~N} \mid$ & IdVZ-Kconomia & 11100 & INIFM & lil Universo \\
\hline & vimern & Ninneroro & Nimero & Nümero & Nimero \\
\hline 1986 & 1 & 0 & () & () & 1 \\
\hline 1987 & 2 & () & () & 0 & 2 \\
\hline 1988 & 7 & () & 2 & () & 9 \\
\hline 1989 & $t$ & () & 8 & 3 & 15 \\
\hline 1990 & 4 & 8 & 7 & 3 & 22 \\
\hline 1991 & 4 & 1 & 1 & 9 & 15 \\
\hline 1992 & 1 & 2 & () & 17 & 20) \\
\hline 1993 & () & 1 & 0 & 6 & 7 \\
\hline Total & 23 & 12 & 18 & $3 x$ & 91 \\
\hline Porcentajje & 25 & 13 & 20 & +2 & 100 \\
\hline
\end{tabular}

Nota: LU\% Ary - Inniversidad del Zulial (Arpuitectura) LuZ Fionomia - Universidad del Zulia (Economia) (JI)() - Universidad de Oriente

UNEIIIM - Inisersidad Experimental lirancisco de Miranda 
Tabla 2 - Principales temas de los trabajos de investigación

\begin{tabular}{|c|c|c|c|c|c|c|}
\hline \multirow[t]{2}{*}{ TEMAS } & LUZZ-Arq. & LUZ-Economia & UID) & UNIEFM & \begin{tabular}{|c|} 
El \\
Universo \\
\end{tabular} & Porcentivil \\
\hline & Numero & Nimero & $\mathrm{N}^{\circ}$ & Número & Nümero & Numern \\
\hline Historia/Turismo & 0 & 1 & 0 & 1 & 2 & 2 \\
\hline Estudio de Factibilidad & 0 & 0 & 1 & 8 & 9 & 10 \\
\hline Comportamiento & 0 & 0 & 2 & 0 & 2 & 2 \\
\hline Mercado & 0 & 0 & 5 & 0 & 5 & 6 \\
\hline Turismo de Eventos & 0 & 0 & 1 & 0 & 1 & 1 \\
\hline Evaluación Recursos & 1 & 1 & 2 & 2 & 6 & 7 \\
\hline Producto T'uristico & 0 & 0 & 1 & 0 & 1 & 1 \\
\hline Planificación & 11 & 4 & 3 & 16 & 34 & 38 \\
\hline Turismo Social & 1 & 0 & 1 & 1 & 3 & 3 \\
\hline Empleo y Formación & 0 & 1 & 2 & 2 & 5 & 6 \\
\hline Circuitos y Rutas & 3 & 0 & 0 & 1 & 4 & 4 \\
\hline Administración & 0 & 0 & 0 & 4 & 4 & 4 \\
\hline Turismo Etnico & 1 & 0 & () & 0 & 1 & 1 \\
\hline Turismo y Desarrollo & 2 & 1 & 0 & 1 & 4 & 4 \\
\hline Organización Turistica & 2 & 1 & 0 & 1 & 4 & 4 \\
\hline Espacio Turistico & 1 & 0 & 0 & 0 & 1 & 1 \\
\hline Corredor Turistico & 1 & 0 & 0 & 0 & 1 & 1 \\
\hline Turismo/Ambiente & 0 & 2 & () & () & 2 & 2 \\
\hline Turismo Rural & 0 & 1 & 0 & 1 & 2 & 2 \\
\hline TOTAL & 23 & 12 & 18 & 38 & 91 & 100 \\
\hline$N^{0}$ de Graduados & 15 & 19 & 18 & 68 & 120 & - \\
\hline Graduados/No lesis & 1,15 & 1,58 & 1,00 & 1,79 & 1,48 & -. \\
\hline
\end{tabular}

Nota: LUZ Arc. - Universidad del Zulia (Arquitectura)

LUZ Economia - Universidad del Lulia (Economía)

UIDO - Universidad de (Oriente

UNEFEM - Universidad Experimental Francisco de Miranda 